\title{
A retrospective study of prevalence and antibiotic resistance of bacteria isolated from the cerebrospinal fluid of neurosurgical patients in a Shanghai tertiary hospital
}

Min Liu

Department of Neurosurgery, Shanghai East Hospital, Tongji University School of Medicine

\section{Yunxia Zhu}

Department of Laboratory Medicine, Shanghai East Hospital, Tongji University School of Medicine

Yin Zhou

Department of Neurosurgery, Shanghai East Hospital, Tongji University School of Medicine

\section{Siyi Xu}

Department of Neurosurgery, Shanghai East Hospital, Tongji University School of Medicine

\section{Jing Ma}

Department of Neurosurgery, Shanghai East Hospital, Tongji University School of Medicine

\section{Tao Zhang}

Department of Neurosurgery, Shanghai East Hospital, Tongji University School of Medicine

\section{Li Xu}

Department of Neurosurgery, Shanghai East Hospital, Tongji University School of Medicine

\section{Wenjuan Wu}

Department of Laboratory of Medicine, Shanghai East Hospital, Tongji University School of Medicine

\section{Chunlong Zhong ( $\nabla$ drchunlongzhong@126.com )}

Department of Neurosurgery, Shanghai East Hospital, Tongji University School of Medicine https://orcid.org/00000002-0605-7273

\section{Research article}

Keywords: postoperative central nervous system infections, neurosurgery, antibiotic resistance, prevalence

Posted Date: February 13th, 2020

DOI: https://doi.org/10.21203/rs.2.23500/v1

License: (c) (i) This work is licensed under a Creative Commons Attribution 4.0 International License. Read Full License 


\section{Abstract}

Background: Postoperative central nervous system infections (PCNSIs) are serious complications following neurosurgery. Effective management of the infections depends on the identification of causative pathogens and the antibiotic sensitivities. The aim of this study was to investigate the clinical features, causative organisms and their antimicrobial susceptibility testing results, which could help clinicians to initiate appropriate empirical antibiotic therapy.

Methods: This was a retrospective study conducted over a period of 3.5 years (from January 2016 to July 2019). All inpatients admitted neurosurgery and ICU meeting the inclusion criteria of PCNSIs were included in the study. Demographic characteristics, type of neurosurgery, laboratory data, causative organisms and antimicrobial susceptibility testing results were analyzed.

Results: A total of 1500 patients underwent operation during this study. Twenty-three patients with 34 isolates out of 79 patients with 157 CSF culture samples were available for data analysis. The estimated incidence and culture-positive rate of PCNSIs were approximately $1.5 \%$ and $29.1 \%$, respectively. The predominant organism was coagulase-negative staphylococci, of which most were methicillin-resistant coagulase-negative staphylococci (MRCoNS). All were susceptible to vancomycin, linezolid, and rifampicin. Acinetobacter baumannii was the most frequent causative Gram-negative agent and was resistant to 14 out of 17 antimicrobials tested. The sensitivity rates for amikacin, sulfamethoxazole-trimethoprim and minocycline were only 44,44 , and $11 \%$, respectively.

Conclusions: The MRCoNS were the predominant organism for PCNSIs. The management of Acinetobacter baumannii could be a major clinical challenge with few effective antimicrobials in PCNSIs.

\section{Background}

Central nervous system infections (CNSIs), which can be caused by a number of different bacteria, viruses, fungi, and parasites, cause substantial disease and death [1]. Cranial operation was considered as a major risk factor for CNSIs. Postoperative central nervous system infections (PCNSIs) are serious complications following neurosurgery, especially craniotomies, resulting in poor prognosis and increasing the total cost of illness [2]. The diagnosis of PCNSIs requires a high degree of clinical suspicion and lumbar puncture for cerebrospinal fluid (CSF) culture. Once diagnosed, selection of the empirical antibiotic should be guided by knowledge of the causative pathogens and their sensitivities.

Although most studies reported that the primary pathogens in PCNSIs are Gram-positive bacteria, such as Staphylococcus aureus, there is a possible trend towards greater proportions of Gram-negative bacteria, especially Acinetobacter species $[3,4]$. There are increased rates of multi-antibiotic resistant strains such as methicillin-resistant S. aureus (MRSA) and strains that produce extended-spectrum $\beta$-lactamases (ESBL) in recent years [5]. It led to increasingly difficult to initiate treatment therapy because of these multi-antibiotic resistant organisms.

Furthermore, few data regarding prevalence and antibiotic resistance of organisms have been available to guide policy on the prevention, diagnosis, and treatment of CNSIs. Antimicrobial susceptibility data among CNS pathogens is important to effectively manage the infections. Shanghai East Hospital is founded in 1920, which is a university general hospital and tertiary referral center in East China. The department of neurosurgery conducts approximately 1,000 operations each year. This study will present the features of pathogens and investigate the clinical characteristics of post-craniotomy PCNSIs in a single institution over the past 3.5 years.

\section{Methods}

\section{Study subjects and data collection}


This was a retrospective study and the data from January 2016 to July 2019 were reviewed. The CSF samples were isolated from in-patients, who were all admitted to the department of neurosurgery and ICU. The data of patients with CSF positive culture isolates were collected from medical records, which included demographic characteristics, types of neurosurgery, laboratory data, causative organisms and antimicrobial susceptibility testing. All patients received $1.0 \mathrm{~g}$ of a first-generation cephalosporin as prophylactic therapy $1 \mathrm{~h}$ before incision and followed by a 72 -h postoperative course.

\section{Inclusion and exclusion criteria}

The positive result of CSF culture was the main inclusion criteria. The isolation of the same species from the same patient within 3 days was regarded as the same isolate and not counted multiple times. All the patients met clinical diagnostic criteria of central nervous infection [6]. Patients with evidence of concomitant chronic meningitis or encephalitis not due to microbial infection were excluded.

\section{Antibiotic susceptibility tests}

We analyzed the results of antibiotic susceptibility based on the Clinical and Laboratory Standards Institution (CLSI) methods [7]. Pathogenic organisms were considered resistant if they were reported as intermediate or resistant to the agents.

\section{Results}

\section{Patients basic information}

A total of 157 CSF culture samples from 79 patients out of 1500 patients undergoing operations were collected from January 2016 to July 2019. Of these, 23 patients with 34 positive isolates were identified and met the inclusion criteria. Table 1 showed the characteristics of 23 patients with positive CSF cultures. The median days of hospitalization and the interval between neurosurgery and infection were 33 and 10 days, respectively. Among these patients, 18 patients were male and 5 were female. Most patients underwent ventricular drainage (39.1\%) and decompressive craniotomy (30.4\%), who were diagnosed with intracranial hemorrhage. The CSF from patients with PCNSIs showed high leukocyte count, high protein levels, but relatively normal glucose level.

\section{Incidence of PCNIs}

The incidence of PCNSIs was approximately 1.5\% (23/1500) and the estimated culture-positive rate of infection was approximately $29.1 \%(23 / 79)$ and 4 patients with 16 isolates were infected with polymicrobial, in which 2 patients with more than two species. The mortality of culture-positive PCNIs was $21.7 \%(5 / 23)$, which was much higher than the mortality of in-patients in general.

\section{Distributions of bacteria isolated}

As table 2 showed, the distribution of Gram-positive, Gram-negative and Eumycetes were 56, 44 and $0 \%$, respectively. The predominant Gram-positive isolate was coagulase-negative staphylococci (38\%), followed by Enterococcus faecalis (6\%). Staphylococcus aureus was not identified in all isolates. Acinetobacter baumannii was the predominant Gram-negative organism and was identified in $10(29 \%)$ isolates, followed by Klebsiella pneumonia in 2 (6\%). Pseudomonas aeruginosa was not found.

\section{Bacterial antibiotic sensitivity}

Table 3 showed the in vitro antibiotic sensitivities of Gram-positive isolates. Antibiotic sensitivities of 13 isolates, which was positive for Coagulase-negative staphylococci, were available for analysis. All these 13 isolates were sensitive to 
linezolid, rifampicin, and vancomycin. Eleven isolates were sensitive to cefoxitin and tigecycline, and 10 isolates were sensitive to gentamycin and teicoplanin. All 13 Coagulase-negative staphylococci positive isolates were resistant to penicillin. Enterococcus faecalis isolates were sensitive to ampicillin, gentamycin, linezolid, penicillin and vacomycin. Those isolates were resistant to erythromycin.

Nine Acinetobacter baumannii isolates were available for analysis. They displayed relative low sensitivity to amikacin and sulfamethoxazole-trimethoprim, as Table 4 showed. Two Klebsiella pneumonia isolates were resistant to all antibiotics, which was used in the test.

\section{Discussion}

PCNSIs were reported to be the most serious complications after craniotomy, with an incidence of varying from $0.9-7.4 \%$, characterized by high incidence, morbidity and mortality [2, 8-11]. In present study, we reported an incidence of PCNSI was $1.5 \%$, which was similar to those previous studies. In our study, only culture-positive patients were included, implying a lower false-positive rate and it might be the possible reason of relative low incidence. The initial symptoms of PCNSIs are difficult to distinguish by different responsible pathogens, such as bacteria, viruses, fungi, and parasites. A retrospective study revealed the bacterial meningitis accounted for more than $86 \%$ (BM) of CNSIs in China [12]. This result was in line with our study, which showed the bacterial infection was the most prevalent subtype.

In table 1, ventricular drainage was the most common operation type in 23 PCNSI patients (9/23, 39.1\%), which indicating that operation type might be a risk factor for the infection. It is important to adopt appropriate operation type, by decreasing operation time, carefully preventing drainage from pollution site and proper treatment could be effective.

Microbial culture and identification remain the gold standard for diagnosing bacterial meningitis [13, 14]. However, with the widespread applications of prophylactic antibiotics, the culture-positive rate of infection was relatively low, reporting from $7.5 \%$ to $50 \%$ [15-17]. In our study, all patients received $1.0 \mathrm{~g}$ cephalosporin as prophylactic therapy, which might lead to the relative lower CSF culture positive rate (29.1\%). In addition, we set the diagnosis standard that the same species of microorganisms cultured from different isolates within 3 days were counted as a single isolate. Hence force, this may be another reason responsible for the low CSF culture positive rate. Moreover, different CNSIs diagnostic standards vary from study to study, which also could contribute to the low culture-positive rate.

Our study demonstrated Gram-positive bacteria was the most common organism, accounting for approximately $56 \%$ of the total isolates. This result was consistent with previous studies [12,18,19]. On the contrary, Aishwarya et al from India reported the proportion of Gram-negative bacteria causing PCNSIs was even as high as 91.6\% [20]. The proportion disparities lie in preoperative therapies, operative aseptic procedures and postoperative managements. However, there does exist a tendency that Gram-negative bacteria increase in prevalence, especially the Acinetobacter baumannii, which was the most frequent causative Gram-negative agent in our study.

Our results showed the coagulase-negative staphylococci were the most prevalent pathogens of Gram-positive bacteria (38\%), which included Staphylococcus epidermidis (12\%), Staphylococcus haemolyticus (6\%), Staphylococcus capitis (9\%), Staphylococcus warneri (6\%) and Staphylococcus hominis (3\%), followed by Enterococcus faecalis (6\%). Twelve (92.3\%) coagulase-negative staphylococci were methicillin-resistant coagulase-negative staphylococci (MRCoNS) and they were susceptible to vancomycin, rifampicin and linezolid. The results were mostly consistent with the results of previous studies $[21,22]$. In order to compare the efficacy and adverse effects of vancomycin versus cefazolin as antimicrobial prophylaxis for CSF shunt infection, E. Tacconelli et al found mortality of patients with post-surgical infections was higher in cefazolin group, implying the use of vancomycin could reduce the infections in the context of high prevalence of methicillin-resistant bacteria [23]. Furthermore, vancomycin was also the last resort and the drug of choice to treat infections caused by Gram-positive bacteria in CNS infection.

Page $4 / 10$ 
Acinetobacter baumannii has emerged as an important nosocomial pathogen causing infections [24]. The SENTRY Antimicrobial Surveillance Program reported that the frequency of Acinetobacter spp. was approximately 7\% [25]. However, there were 10 (29\%) Acinetobacter baumannii isolates in our study, and all isolates were multidrug-resistant (MDR) bacteria with resistance to 14 out of 17 antimicrobials tested. The global antimicrobial surveillance study and the Tigecycline Evaluation and Surveillance Trial (T.E.S.T) showed that meropenem resistant Acinetobacter spp. increased from $17.7 \%$ to $33.0 \%$ and multi-drug resistant Acinetobacter spp. increased from $25.6 \%$ to $49.7 \%$ in $2005-2007$ to 2008 2012 [26], and the rate of carbapenem-resistant Acinetobacter baumannii (CRAB) has risen in recent years [27]. The emergence of MDR Acinetobacter baumannii, known as one of the ESKAPE (Enterococcus faecium, Staphylococcus aureus, Klebsiella pneumoniae, A. baumannii, Pseudomonas aeruginosa, and Enterobacter species) pathogens [28], has become a serious medical problem worldwide $[29,30]$. Acinetobacter baumannii has many resistance mechanisms, including ß-lactamases, aminoglycoside-modifying enzymes, efflux pumps, permeability defects, and modifications of target sites. The accumulation of several resistance mechanisms in A. baumannii has gradually decreased the number of antibiotic classes available to treat Acinetobacter baumannii infections in clinical practice. To combat the MDR Acinetobacter baumannii, combination therapies including colistin/imipenem, colistin/meropenem, colistin/rifampicin, colistin/tigecycline, colistin/sulbactam, colistin/teicoplanin, and imipenem/sulbactam should be treated. In addition, new non- antibiotic methods such as bacteriophage, has renewed interest in the therapy [31].

Klebsiella pneumonia is an opportunistic pathogen capable of causing a variety of infections, and we found two isolates $(6 \%)$ in our study. The infection incidence differs from place to place and with different age groups [32,33]. The percentage were: 96 (68\%) of 142 in Taiwan, 25 (43\%) of 68 in the United States, 28 (39\%) of 71 in Australia, 40 (34\%) of 116 in South Africa, 6 (22\%) of 27 in Europe, and 7 (17\%) of 41 in Argentina [33]. Alarmingly, multidrug-resistant of Klebsiella pneumonia was detected among the Gram-negative bacteria. Previous studies reported Klebsiella pneumonia have acquired antimicrobial resistance genes, and these strains encoded two plasmids with drug-resistance potential $[34,35]$. Widespread, highly strains armed with multidrug-resistant determinants are a great cause for concern. Changes of local microenvironments and combinations of antibiotics may improve the treatment efficacy.

The mortality rate of PCNSIs dropped drastically in past years, from 34\% in 2005 [36] to 19\% in 2011 [37], and a recent study reported that the mortality was 3.6\% [16]. The mortality of present study was relatively higher than those reported, which was $21.7 \%$. It could be explained by that only 23 patients were included by our restrict inclusion criteria and more data are required. Another possible explanation might be that the diagnosis of PCNSIs in this study depended on the isolation of a culture results rather than clinical signs, so that many infected patients without positive cultures might not be included. Therefore, the incidence, positive-culture rate, mortality and pathogens of PCNSIs vary between studies and are difficult to compare between different studies.

The significance of this study not only showed the distribution of pathogens from a region, but also reflected a similar trend to that observed in China. Clinicians would choose the appropriate empirical antibiotic therapy for PCNSIs according to the characteristics of the pathogenetic organisms. There are also several limitations. Firstly, the number of samples was not large enough to represent the whole situation of China. Secondly, we had no data for the analyses of Eumycetes, tuberculous and parasitic infections. Furthermore, the mechanisms of drug-resistance were not explored because of molecular detection was not performed. Notwithstanding its limitations, this study does provide an outline of bacterial prevalence of PCNSIs and the study continues to update data.

\section{Conclusions}

Postoperative central nervous system infections (PCNSIs) are serious complications and usually led to a higher mortality rate. MRCoNS was the predominant organism isolated and was totally susceptible to vancomycin, linezolid, and 
rifampicin. The management of Acinetobacter baumannii remains a major clinical challenge with high mortality and few effective antimicrobials were available in the treatment of PCNSIs.

\section{Abbreviations}

CNSIs: central nervous system infections; PCNSIs: postoperative central nervous system infections; CSF: cerebrospinal fluid; MRSA: methicillin-resistant S. aureus; ESBL: extended-spectrum $\beta$-lactamases; CLSI: Clinical and Laboratory Standards Institution; MRCoNS: methicillin-resistant coagulase-negative staphylococci; MDR: multidrug-resistant; T.E.S.T: Tigecycline Evaluation and Surveillance Trial; CRAB: carbapenem-resistant Acinetobacter baumannii.

\section{Declarations}

\section{Acknowledgement}

Not applicable.

Authors' contributions: CZ, WW and LX conceived the study and provided data interpretation. ML and YZ analyzed the data and drafted the manuscript. YXZ provided expert microbiology analysis and interpretation of study data. JM, TZ and SX provided clinical expertise in interpretation of data and drafting manuscript. All authors read and approved the final manuscript.

Funding: This study was supported by grants from the National Natural Science Foundation of China (Nos. 81771332, 81571184, both to CZ; 81600625, to ML); the Shanghai Key Medical Discipline for Critical Care Medicine of China (No. 2017zz02017, to CZ); the Key Discipline Construction Project of Pudong Health Bureau of Shanghai (No. PWZxk2017-23, to CZ), the Top-level Clinical Discipline Project of Shanghai Pudong (No. PWYgf2018-05, to CZ) and the Outstanding Leaders Training Program of Pudong Health Bureau of Shanghai of China (No. PWR12018-07, to CZ).

Availability of data and materials: The data used and/or analyzed in this study are available from the corresponding authors on reasonable request.

Ethics approval and consent to participate: This study was approved by the Ethics Board of Shanghai East Hospital, Tongji University School of Medicine in China. Written Informed consent for this study was obtained from all patients or their family members.

\section{Consent for publication}

Not applicable.

\section{Competing interests}

Professor Chunlong Zhong is an associate editor for BMC Neurology. The authors declare that they have no competing interests.

\section{References}

1. Dubot-Pérès A, Mayxay M, Phetsouvanh R, Lee SJ, Rattanavong S, Vongsouvath M, Davong V, Chansamouth V, Phommason K, Moore C, et al. Management of Central Nervous System Infections, Vientiane, Laos, 2003-2011. Emerg Infect Dis. 2019; 25(5):898-910.

2. Ma YF, Wen L, Zhu Y. Prospective study evaluating post-operative central nervous system infections following cranial surgery. Br J Neurosurg. 2019; 33(1):80-83. 
3. Tangden T, Enblad P, Ullberg M, Sjolin J. Neurosurgical Gram-Negative Bacillary Ventriculitis and Meningitis: A Retrospective Study Evaluating the Efficacy of Intraventricular Gentamicin Therapy in 31 Consecutive Cases. Clinical Infectious Diseases, 2011; 52(11):1310-1316.

4. Guardado, Rodríguez, Asensi V, Torres JM, Perez F, Blanco A, Maradona JA, Carton JA. Post-surgical enterococcal meningitis: Clinical and epidemiological study of 20 cases. Scand J Infect Dis. 2006; 38(8):584-588.

5. Spatenkova V, Bradac O, Fackova D, Bohunova Z, Suchomel P. Low incidence of multidrug-resistant bacteria and nosocomial infection due to a preventive multimodal nosocomial infection control: a 10-year single centre prospective cohort study in neurocritical care. BMC Neurol. 2018; 18(1):23.

6. Tunkel A R, Hasbun R, Bhimraj A, Byers K, Kaplan SL, Scheld WM, van de Beek D, Bleck TP, Garton HJL, Zunt JR. 2017 Infectious Diseases Society of America's Clinical Practice Guidelines for Healthcare-Associated Ventriculitis and Meningitis. Clin Infect Dis. 2017; 64(6): e34-e65.

7. CLSI. Performance standards for antimicrobial susceptibility testing. 29th ed. CLSI supplement M100. Wayne, PA: Clinical and Laboratory Standards Institute; 2019.

8. O"Keeffe A B, Lawrence T, Bojanic S. Oxford craniotomy infections database: A cost analysis of craniotomy infection[J]. Br J Neurosurg. 2012; 26(2):265-269.

9. Korinek A M, Golmard J L, Elcheick A, Bismuth R, van Effenterre R, Coriat P, Puybasset L. Risk factors for neurosurgical site infections after craniotomy: a critical reappraisal of antibiotic prophylaxis on 4578 patients. $\mathrm{Br} \mathrm{J}$ Neurosurg. 2005; 19(2):155-162.

10. Link-Gelles R, Toews K A, Schaffner W, Edwards KM, Wright C, Beall B, Barnes B, Jewell B, Harrison LH, Kirley PD. Characteristics of Intracranial Group A Streptococcal Infections in US Children, 1997-2014. J Pediatric Infect Dis Soc. 2018.

11. Papavasileiou K, Papavasileiou E, Tzanakaki G, Voystzi A, Kremastinou J, Chatzipanagiotou S. Acute bacterial meningitis cases diagnosed by culture and PCR in a children's hospital throughout a 9-Year period (2000-2008) in Athens, Greece. Mol Diagn Ther. 2011; 15(2):109-13.

12. Tian L, Zhang Z, Sun ZY. Pathogen Analysis of Central Nervous System Infections in a Chinese Teaching Hospital from 2012-2018: A Laboratory-based Retrospective Study. Curr Med Sci. 2019; 39(3):449-454.

13. Zhang X, Medow JE, Iskandar BJ, Wang F, Shokoueinejad M, Koueik J, Webster JG. Invasive and noninvasive means of measuring intracranial pressure: A review. Physiol Meas. 2017; 38(8):R143-182.

14. Nag DS, Sahu S, Swain A, Kant S. Intracranial pressure monitoring: Gold standard and recent innovations. World J Clin Cases. 2019; 7(13):1535-1553.

15. Du B, Hua C, Xia Y, Li J, Xie Y, Tao Y, Cao Q, Mo X. Evaluation of the BioFire FilmArray meningitis/encephalitis panel for the detection of bacteria and yeast in Chinese children. Ann Transl Med. 2019; 7(18):437.

16. Shi ZH, Xu M, Wang YZ, Luo XY, Chen GQ, Wang X, Wang T, Tang MZ, Zhou JX. Post-craniotomy intracranial infection in patients with brain tumors: A retrospective analysis of 5723 consecutive patients. Br J Neurosurg. 2017; 31:5-9.

17. Kourbeti IS, Jacobs AV, Koslow M, Karabetsos D, Holzman RS. Risk factors associated with postcraniotomy meningitis. Neurosurgery. 2007; 60(2):317-25.

18. Tian R, Hao S, Hou Z, Gao Z, Liu B. The Characteristics of Post-neurosurgical Bacterial Meningitis in Elective Neurosurgery in 2012: A Single Institute Study. Clin Neurol Neurosurg. 2015; 139:41-5.

19. Hang JB, Wu H, Wang H, MA BT, Wang RZ, Wei JJ. Prevalence and antibiotic resistance of bacteria isolated from the cerebrospinal fluid of neurosurgical patients at Peking Union Medical College Hospital. Antimicrob Resist Infect Control. 2018; 7:41-46.

20. Govindaswamy A, Bajpai V, Singh P, Lohiya A, Ayyanar M, Gupta DK, Bindra A, Singh GP, Mathur P. Prevalence and Antibiotic Resistance Profile of Cerebrospinal Fluid Pathogens from Neurosurgical Patients from Level 1 Trauma 
Center in India. Asian J Neurosurg. 2019; 14(3):834-838.

21. Nahaei M R, Shahmohammadi M R, Ebrahimi S, Ebrahimi S, Milani M. Detection of Methicillin-Resistant CoagulaseNegative Staphylococci and Surveillance of Antibacterial Resistance in a Multi-Center Study from Iran. Jundishapur J Microbiol. 2015; 8(8): e1945.

22. Liesse JM, Wambale JM, Lukukula CM, za Balega Takaisi-Kikuni N. High prevalence of methicillin resistant staphylococci strains isolated from surgical site infections in Kinshasa. Pan Afr Med J. 2014; 18:322.

23. Tacconelli E, Cataldo MA, Albanese A, Tumbarello M, Arduini E, Spanu T, Fadda G, Anile C, Maira G, Federico G, Cauda R. Vancomycin versus cefazolin prophylaxis for cerebrospinal shunt placement in a hospital with a high prevalence of meticillin-resistant Staphylococcus aureus. J Hosp Infect. 2008; 69(4):337-344.

24. Peleg A Y, Seifert H, Paterson D L. Acinetobacter baumannii: Emergence of a Successful Pathogen. Clin Microbiol Rev. 2008; 21(3):538-82.

25. Sader HS, Farrell DJ, Flamm RK, Jones RN. Antimicrobial susceptibility of Gram-negative organisms isolated from patients hospitalised with pneumonia in US and European hospitals: Results from the SENTRY Antimicrobial Surveillance Program, 2009-2012. Int J Antimicrob Agents. 2014; 43(4):328-334.

26. Morfin-Otero R, Noriega ER, Dowzicky MJ. Antimicrobial susceptibility trends among gram-positive and -negative clinical isolates collected between 2005 and 2012 in Mexico: results from the Tigecycline Evaluation and Surveillance Trial. Ann Clin Microbiol Antimicrob. 2015; 14:53.

27. Guo J, Li C. Molecular epidemiology and decreased susceptibility to disinfectants in carbapenem-resistant Acinetobacter baumannii isolated from intensive care unit patients in central China. J Infect Public Health. 2019; 12(6):890-896.

28. Boucher H, Talbot G, Bradley J, Edwards J, Gilbert D, Rice L, Scheld M, Spellberg B, Bartlett J. Bad Bugs, No Drugs: No ESKAPE! An Update from the Infectious Diseases Society of America. Clin Infect Dis. 2009; 48(1):1-12.

29. Shrestha S, Tada T, Miyoshi-Akiyama T, Ohara H, Shimada K, Satou K, Teruya K, Nakano K, Shiroma A, Sherchand JB, et al. Molecular epidemiology of multidrug-resistant Acinetobacter baumannii isolates in a university hospital in Nepal reveals the emergence of a novel epidemic clonal lineage. Int J Antimicrob Agents. 2015; 46(5):526-31.

30. Duarte A, Ferreira S, Almeida S, Domingues FC. Clinical isolates of Acinetobacter baumannii from a Portuguese hospital: PFGE characterization, antibiotic susceptibility and biofilm-forming ability. Comp Immunol Microbiol Infect Dis. 2016; 45:29-33.

31. Lee CR, Lee JH, Park M, Park KS, Bae IK, Kim YB, Cha CJ, Jeong BC, Lee SH. Biology of Acinetobacter baumannii: Pathogenesis, Antibiotic Resistance Mechanisms, and Prospective Treatment Options. Front Cell Infect Microbiol. 2017; 7:55.

32. Carrie C, Walewski V, Levy C, Alexandre C, Baleine J, Charreton C, Coche-Monier B, Caeymaex L. Klebsiella pneumoniae and Klebsiella oxytoca meningitis in infants. Epidemiological and clinical features. Arch Pediatr. 2019; 26(1):12-15.

33. Ko WC, Paterson DL, Sagnimeni AJ, Hansen DS, Von Gottberg A, Mohapatra S, Casellas JM, Goossens H. Community-Acquired Klebsiella pneumoniae Bacteremia: Global Differences in Clinical Patterns. Emerg Infect Dis. 2002; 8(2):160-6.

34. Karlsson M, Stanton RA, Ansari U, McAllister G, Chan MY, Sula E, Grass JE, Duffy N, Anacker ML. Identification of a Carbapenemase-Producing Hypervirulent Klebsiella pneumoniae Isolate in the United States. Antimicrob Agents Chemother. 2019; 63(7): e00519-19.

35. Holt KE, Wertheim H, Zadoks RN, Baker S, Whitehouse CA, Dance D, Jenney A, Connor TR, Hsu LY. Genomic analysis of diversity, population structure, virulence, and antimicrobial resistance in Klebsiella pneumoniae, an urgent threat to public health. Proc Natl Acad Sci U S A. 2015; 112(27): E3574-3581. 
36. Wang KW, Chang WN, Huang CR, Tsai NW, Wang HC, Su TM, Rau CS, Cheng BC, Chang CS. Post-neurosurgical nosocomial bacterial meningitis in adults: microbiology, clinical features, and outcomes. J Clin Neurosci. 2005; 12(6):647-650.

37. Tangden T, Enblad P, Ullberg M, Sjolin J. Neurosurgical Gram-Negative Bacillary Ventriculitis and Meningitis: A Retrospective Study Evaluating the Efficacy of Intraventricular Gentamicin Therapy in 31 Consecutive Cases. Clin Infect Dis. 2011; 52(11):1310-1316.

\section{Tables}

Table 1 Demographic and clinical characteristics of 23 patients with bacterial meningitis

\begin{tabular}{ll}
\hline Characteristic & Value \\
\hline $\begin{array}{l}\text { Demographic parameters } \\
\text { Mean age (yr) }\end{array}$ & 58.8 \\
\hline Sex, male:female & $18: 5$ \\
\hline Interval between neurosurgery and infection (median day) & $10\left(\mathrm{IQR}^{\mathrm{a}}=17\right)$ \\
\hline Days in hospital (median) & $33(\mathrm{IQR}=29)$ \\
\hline Death & $5 \square 21.7 \% \square$ \\
\hline Operation types & \\
\hline Craniotomy & $6(26.1 \%)$ \\
\hline Ventricular drainage & $9(39.1 \%)$ \\
\hline Decompressive craniotomy & $7(30.4 \%)$ \\
\hline Intracranial artery embolization & $1(4.4 \%)$ \\
\hline CSF data & \\
\hline Leukocyte count (10^6/L) & $63(\mathrm{IQR}=344)$ \\
\hline Glucose level (mmol/L) & $3.48(\mathrm{IQR}=2.565)$ \\
\hline Protein level (g/L) & $1.16(\mathrm{IQR}=2.6)$ \\
\hline
\end{tabular}

${ }^{\mathrm{a} I Q R}$ interquartile range

Table 2 Bacteria isolated from cerebrospinal fluid $(\mathrm{N}=34)$

\begin{tabular}{|c|c|}
\hline Organism & $\mathrm{n}(\%)$ \\
\hline Gram-positive bacteria & $19(56)$ \\
\hline Coagulase-negative staphylococci ${ }^{\mathrm{a}}$ & $13(38)$ \\
\hline Enterococcus faecalis & $2(6)$ \\
\hline Staphylococcus aureus & $0(0)$ \\
\hline Others $^{b}$ & $4(12)$ \\
\hline Gram-negative bacteria & $15(44)$ \\
\hline Acinetobacter baumannii & $10(29)$ \\
\hline Klebsiella pneumoniae & $2(6)$ \\
\hline Pseudomonas aeruginosa & $0(0)$ \\
\hline Others $^{\mathrm{c}}$ & $3(9)$ \\
\hline Eumycetes & $0(0)$ \\
\hline
\end{tabular}

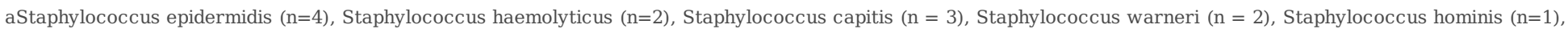

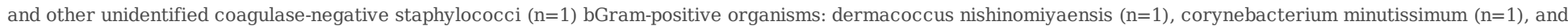

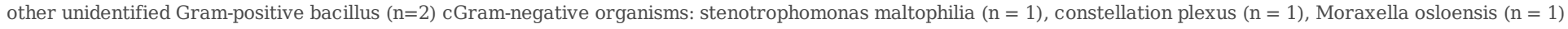

Table 3 Gram-positive antibiotic sensitivity

\begin{tabular}{|c|c|c|c|c|c|c|c|c|c|c|c|c|c|c|c|c|c|c|c|}
\hline Organism & AMP & CIP & CLI & ERY & FOS & FOX & GEN & LNZ & LVX & $\mathrm{MNO}$ & OXA & PEN & RIF & $\mathrm{SM}$ & SXT & TCY & TEC & TGC & VAN \\
\hline $\begin{array}{l}\text { Coagulase-negative } \\
\text { staphylococci [13] }\end{array}$ & 1 & 5 & 6 & 4 & 4 & 11 & 10 & 13 & 5 & 9 & 1 & 0 & 13 & 1 & 9 & 7 & 10 & 11 & 13 \\
\hline $\begin{array}{l}\text { Enterococcus faecalis } \\
\text { [2] }\end{array}$ & 2 & 1 & I & 0 & I & I & 2 & 2 & 1 & 1 & I & 2 & I & 1 & I & 1 & I & 1 & 2 \\
\hline
\end{tabular}

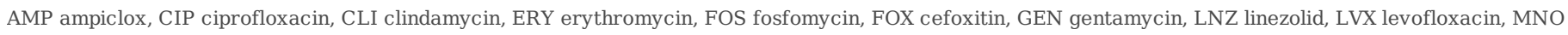

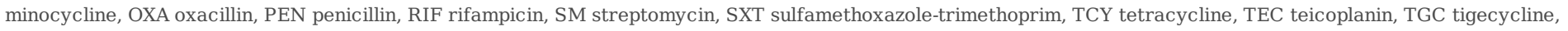


VAN vancomycin

Table 4 Gram-negative antibiotic sensitivity

\begin{tabular}{|c|c|c|c|c|c|c|c|c|c|c|c|c|c|c|c|c|c|c|c|c|}
\hline Organism & AMK & ATM & CAZ & CIP & $\mathrm{CRO}$ & CSL & CTX & FEP & FOS & GEN & IPM & LVX & MEM & MNO & PIP & SAM & SXT & TOB & TGC & $\mathrm{PB}$ \\
\hline $\begin{array}{l}\text { Acinetobacter } \\
\text { baumannii [9] }\end{array}$ & 4 & 1 & 0 & 0 & 0 & 0 & 1 & 0 & 1 & 0 & 0 & 0 & 0 & 1 & 0 & 0 & 4 & 0 & 0 & 0 \\
\hline $\begin{array}{l}\text { Klebsiella } \\
\text { pneumoniae } \\
{[2]}\end{array}$ & I & 0 & 0 & 0 & 0 & 0 & 0 & 0 & 0 & 0 & 0 & 0 & 1 & 1 & I & 0 & 0 & 0 & 0 & I \\
\hline
\end{tabular}

AMK amikacin, ATM aztreonam, CAZ ceftazidime, CIP ciprofloxacin, CRO ceftriaxone, CSL cefoperazone-sulbactam, CTX cefotaxime, FEP cefepime, FOS

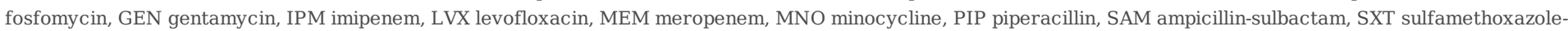
trimethoprim, TOB tobramycin, TGC tigecycline, PB polymyxin 